\title{
Delayed Restricted Diffusion in Carbon Monoxide Leukoencephalopathy
}

\author{
Madeleine E. Sharp, Jason B. Chew, Manraj K.S. Heran, Jacqueline A. Pettersen
}

Can J Neurol Sci. 2012; 39: 393-394

In the acute phase, carbon monoxide $(\mathrm{CO})$ poisoning can lead to a wide range of neurological symptoms, ranging from headaches, dizziness and altered mental status to coma. The basis for this is thought to be hypoxia. Carbon monoxide competes with oxygen for binding to hemoglobin $(\mathrm{Hb})$ and once bound, shifts the oxyhemoglobin dissociation curve to the left, thus impairing off-loading of oxygen molecules, leading to hypoxia .

A sizable minority of $\mathrm{CO}$ poisoning cases also develop a delayed neuropsychiatric syndrome (DNS) following a lucid interval of 2-40 days. The underlying pathophysiology of DNS is much less well understood and only recently, with the advent of advanced neuroimaging techniques, have we been able to draw distinctions between DNS and other forms of cellular injury ${ }^{1}$. We present a case of delayed neuropsychiatric syndrome following CO poisoning for whom diffusion weighted imaging (DWI) magnetic resonance imaging (MRI) revealed restricted diffusion but in a temporal pattern distinct from ischemia.

\section{Case Report}

A 51-year-old man was admitted with acute $\mathrm{CO}$ poisoning (COHb level: $37 \%$ ). He was obtunded and required admission to the intensive care unit. Computed tomogram (CT) revealed diffuse low attenuation changes within the cerebral hemispheric white matter (WM), as well as focal low attenuation within the globi pallidi (Figure 1a). A urine toxicology screen was negative for any alternative or concurrent toxic exposures. He improved steadily following normobaric oxygen therapy and mechanical ventilation, and was transferred to the ward one week later with a grossly normal mental status and neurological examination.

By day 15 post-intoxication, he had developed akinetic mutism, incontinence, and required enteral feeding. The CT showed progressive worsening of the diffuse subcortical white matter hypoattenuation changes (Figure, a-c), and MRI at five weeks post-intoxication revealed extensive T2 hyperintensity affecting the cerebral hemispheric WM, mimicking changes as seen with diffuse small vessel ischemic change. However, these changes were associated with restricted diffusion (Figure, d-e), consistent with ongoing cytotoxic injury. Following this acute deterioration, he gradually improved in all symptoms but required inpatient rehabilitation for five months. Neuropsychological assessment at six months post-exposure revealed deficits in attention, executive functioning, visuospatial processing and short-term memory. He scored 19/30 on the Mini-Mental Status Exam (MMSE) and 52/114 on the Behavioural Neurology Assessment (BNA), far below what would be considered normal for age and education level. Neurological exam revealed flat affect, Parkinsonian gait,

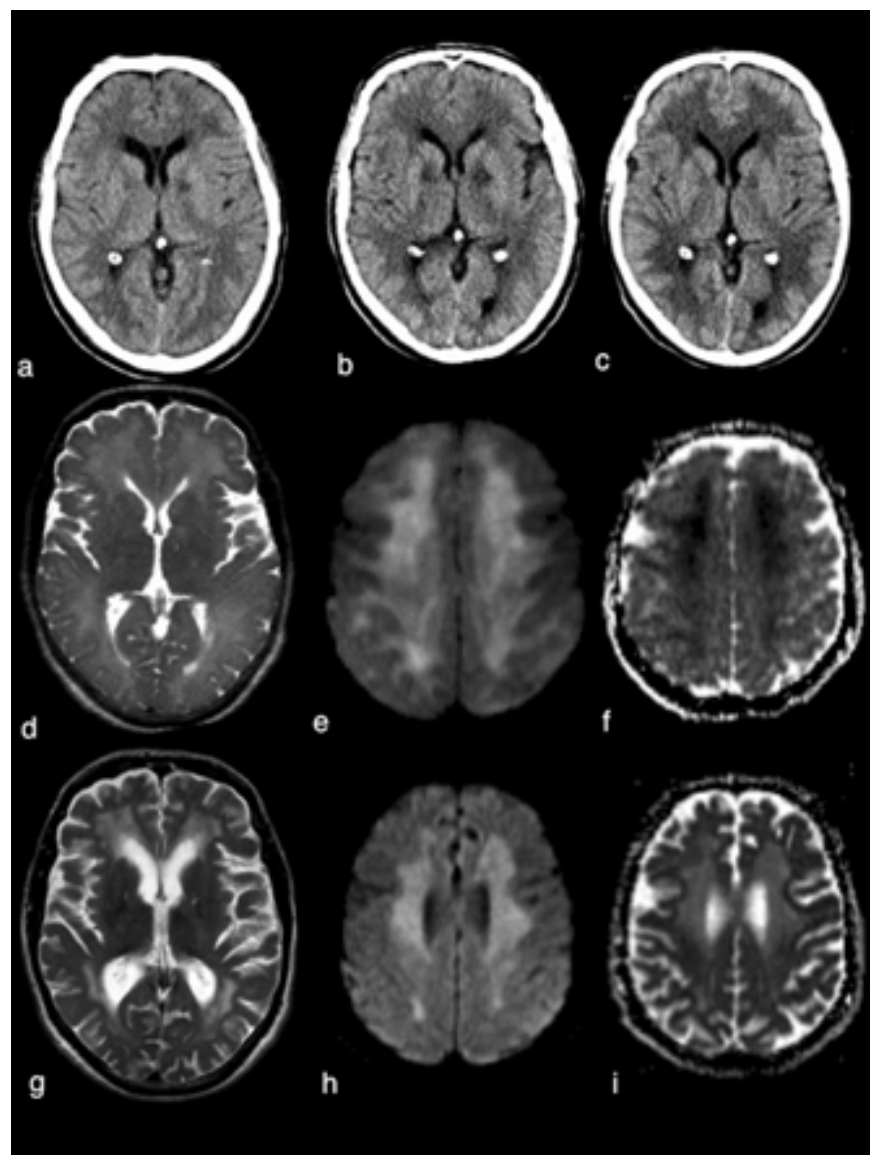

Figure: Temporal evolution of imaging findings on CT and MRI. a-c: Serial unenhanced axial CT head at (a) 5 hours, (b) 3 days and (c) 1 month following acute $\mathrm{CO}$ intoxication demonstrates focal hypoattentuation within the globi pallidi and progressive worsening of diffuse cerebral hemispheric white matter low-attenuation changes. $d$-f: Axial (d) T2 MRI, (e) b1000 DWI and (f) apparent diffusion coefficient $(A D C)$ map at 5 weeks post-intoxication reveals increased signal intensity, and restricted diffusion, respectively, within the affected white matter. g-i: Axial ( $g$ ) T2 MRI, (h) b1000 DWI and (i) ADC map at 1 year reveal persisting increased signal within the white matter, and resolution of previously documented restricted diffusion, with generalized cerebral atrophy.

From the Department of Medicine, Division of Neurology (MES, JAP), Department of Radiology (JBC, MKSH), the Northern Medical Program (JAP), University of British Columbia, Vancouver, BC.

Received September 16, 2011. Final Revisions Submitted November 22, 2011 Correspondence to: Madeleine Sharp, Room 8219, 8th Floor, Gordon \& Leslie Diamond Health Care Centre, 2775 Laurel Street, Vancouver, British Columbia, V5Z 1M9, Canada. Email: madeleinesharp@gmail.com 
rigidity and tremor. He had poor insight into his disability, required a walker, and was limited in his activities of daily living as a result of impaired mobility.

At ten months, his Parkinsonian gait and incontinence had largely resolved and there were mild improvements in all neuropsychological domains, most notably visuospatial and executive functioning. He scored $21 / 30$ on the MMSE and $73 / 114$ on the BNA. Repeat MRI revealed persistent diffuse T2 hyperintensities throughout the WM, without restricted diffusion, and extensive cerebral atrophy (Figure, g-i).

\section{Discussion}

The progressive development of white matter signal changes, including restricted diffusion, on MRI during the development of DNS suggests an underlying cytotoxic process ${ }^{1}$ within the WM, with ischemia being a potential contributor. However, the protracted time course of these changes (i.e., at least three weeks) would be unusual for ischemia, in which restricted diffusion typically resolves within seven to ten days of the ischemic event. Further, these changes on MRI and the concomitant clinical manifestations began two weeks following the hypoxic insult, suggesting that the initial hypoxic insult was not directly responsible for DNS. These observations lend further support to the contention that the primary pathophysiologic correlate of DNS is not ischemic but, rather, an alternative process such as demyelination ${ }^{1}$, severe enough in this case to result in generalized atrophy (Figure, g). Indeed, Prockop $^{2}$ hypothesized that lipid peroxidation occurring in the white matter as a result of mitochondrial dysfunction and free radical toxicity might explain the late development of demyelination. Serial magnetic resonance spectroscopy in DNS supports this hypothesis by demonstrating a slowly rising choline concentration, an indicator of myelin breakdown, which peaks at the time of DNS onset and correlates with maximal restricted diffusion on MRI ${ }^{1}$.

Restricted diffusion has been previously documented after the onset of DNS using $\mathrm{DWI}^{3}$ but without neuropsychological correlation. While reduced diffusion has also been demonstrated using diffusion tensor imaging (DTI) either at a single time point ${ }^{4}$ or on serial imaging, neuropsychological correlation has either been limited to the MMSE ${ }^{5,6}$ or tests assessing select cognitive domains at only one point in time ${ }^{4}$. As far as we know, no previous report has correlated the neurocognitive and imaging improvements following DNS using detailed neuropsychological assessment tools and the much more accessible DWI technology. Though DTI is more sensitive than DWI for measuring diffusivity, it is much less accessible and requires expert analysis for interpretation thereby significantly limiting its utility in general neurology practice. In summary, confluent WM T2-hyperintensities underlying CO delayed neuropsychiatric syndrome may appear similar to small vessel ischemic disease on MRI. However, further confirming what has previously been shown using DTI, DWI reveals delayed and prolonged restricted diffusion which helps differentiate this entity from ischemia, and likely reflects a CO-initiated delayed leukoencephalopathy. We have shown that DWI can be useful for demonstrating the evolution of white matter injury in DNS and that the resolution of restricted diffusion reflects improvements on neuro-psychological testing. We recommend
DWI imaging in cases where the clinical history is uncertain and the etiology of white matter change is unclear. Currently, there are no effective forms of prevention or treatment for DNS following $\mathrm{CO}$ exposure. By further elucidating the underlying pathophysiology of this entity through imaging and pathological studies, it is hoped that such therapy will ultimately evolve.

\section{REFERENCES}

1. Murata T, Kimura H, Kado $\mathrm{H}$, et al. Neuronal damage in the interval form of co poisoning determined by serial diffusion weighted magnetic resonance imaging plus $1 \mathrm{~h}$-magnetic resonance spectroscopy. J Neurol Neurosurg Psychiatr. 2001;71 (2):250-3.

2. Prockop LD. Carbon monoxide brain toxicity: clinical, magnetic resonance imaging, magnetic resonance spectroscopy, and neuropsychological effects in 9 people. J Neuroimaging. 2005; 15(2):144-9.

3. Kinoshita T, Sugihara S, Matsusue E, Fujii S, Ametani M, Ogawa T. Pallidoreticular damage in acute carbon monoxide poisoning: diffusion-weighted MR imaging findings. AJNR Am J Neuroradiol. 2005;26(7):1845-8.

4. Lin $\mathrm{W}-\mathrm{C}, \mathrm{Lu} \mathrm{C}-\mathrm{H}$, Lee $\mathrm{Y}-\mathrm{C}$, et al. White matter damage in carbon monoxide intoxication assessed in vivo using diffusion tensor MR imaging. AJNR Am J Neuroradiol. 2009;30(6):1248-55.

5. Terajima K, Igarashi $H$, Hirose $M$, Matsuzawa $H$, Nishizawa $M$, Nakada T. Serial assessments of delayed encephalopathy after carbon monoxide poisoning using magnetic resonance spectroscopy and diffusion tensor imaging on 3.0t system. Eur Neurol. 2008;59(1-2):55-61.

6. Kuroda H, Fujihara K, Takahashi S, Shinozawa Y, Itoyama Y. A case of delayed encephalopathy after carbon monoxide poisoning longitudinally monitored by diffusion tensor imaging. AJNR Am J Neuroradiol. Epub 2011 Aug 18. 